\section{(- OPEN ACCESS}

\title{
Shorter time until return of spontaneous circulation is the only independent factor for a good neurological outcome in patients with postcardiac arrest syndrome
}

\author{
Tomohide Komatsu, Kosaku Kinoshita, Atsushi Sakurai, Takashi Moriya, \\ Junko Yamaguchi, Atsunori Sugita, Rikimaru Kogawa, Katsuhisa Tanjoh
}

Division of Emergency and Critical Care Medicine, Department of Acute Medicine, Nihon University School of Medicine, Itabashi-ku, Tokyo, Japan

\section{Correspondence to} Dr Atsushi Sakurai, Division of Emergency and Critical Care Medicine, Department of Acute Medicine, Nihon University, School of Medicine, 30-1 Oyaguchi Kamimachi Itabashiku, Tokyo 173-8610, Japan; sakurai.atsushi@nihon-u.ac.jp

Received 4 February 2013 Revised 22 March 2013 Accepted 1 April 2013 Published Online First 2 May 2013

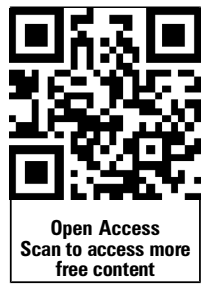

ABSTRACT

Objective Few studies have reported factors that result in a better neurological outcome in patients with postcardiac arrest syndrome (PCAS) following return of spontaneous circulation (ROSC). We investigated the factors affecting neurological outcome in terms of both prehospital care and treatments after arrival at hospital in patients with PCAS.

Methods The study enrolled patients with cardiogenic cardiac arrest who were admitted to an intensive care unit after ROSC with PCAS. We investigated the association of the following factors with outcome: age, gender, witness to event present, bystander cardiopulmonary resuscitation (CPR) performed, ECG waveform at the scene, time interval from receipt of call to arrival of emergency personnel, time interval from receipt of call to arrival at hospital, prehospital defibrillation performed, special procedures performed by emergency medical technician, and time interval from receipt of call to ROSC, coronary angiography/ percutaneous coronary intervention (PCI) and therapeutic hypothermia performed.

Results The study enrolled 227 patients with PCAS. Compared with the poor neurological outcome group, the good neurological outcome group had a statistically significant higher proportion of the following factors: younger age, male, witness present, bystander CPR performed, first ECG showed ventricular fibrillation/ pulseless ventricular tachycardia, defibrillation performed during transportation, short time interval from receipt of call to ROSC, coronary angiography/PCI and therapeutic hypothermia performed. Of these factors, the only independent factor associated with good neurological outcome was the short time interval from receipt of the call to ROSC.

Conclusions In the present study, shortening time interval from receipt of call to ROSC was the only important independent factor to achieve good neurological outcome in patients with PCAS.

\section{INTRODUCTION}

The term, postcardiac arrest syndrome (PCAS), describes organ damage from whole-body ischaemia and reperfusion after return of spontaneous circulation (ROSC) caused by cardiac arrest (CA). Mortality rates from PCAS $^{1} 2$ differ by country, region and facility. ${ }^{3}$ In the west, in-hospital mortality rates after transfer to hospital are $60-70 \% .{ }^{45}$ In Japan, patients are given cardiopulmonary resuscitation (CPR) and transported to hospital even if a long time has passed since the CA, so in-hospital mortality rates are as high as around $90 \%{ }^{6}$
A recent study has reported the following factors related to poor outcome with PCAS: (1) myoclonic status within $24 \mathrm{~h}$ of resuscitation or during three-day observation, (2) loss of light reflex or corneal reflex and (3) no motor response or extension in response to pain. ${ }^{7}$ All these factors were at $24 \mathrm{~h}$ after ROSC and were outcome predictors for survival or mortality. PCAS has high in-hospital mortality rates, so a key challenge from both a medical and social perspective is the clarification of factors to predict good outcomes at an early stage, rather than simply focusing on factors relating to survival and mortality immediately after ROSC in patients with PCAS.

A smooth transition to life support ${ }^{8} 9$ is important if outcome in patients with out-of-hospital CA is to be improved. For CPR, transition to life support starts when an individual is found to have suddenly collapsed or to be unresponsive, immediately raising the suspicion of CA. Once the possibility of CA arises, the emergency services are contacted for help (119; emergency call number in Japan) and efforts are made to ensure the rapid arrival of specialists or emergency personnel bringing resuscitation equipment and an automated external defibrillator (AED). Such equipment is used to perform basic life support (BLS) rapidly, followed by advanced life support (ALS). After ROSC, the patient must receive intensive care at a specialist institution as necessary, which should increase the likelihood of good outcome. ${ }^{10}$ Good neurological outcome must be increased with successful achievement of the chain of survival, that is, rapid contact to the emergency services, rapid start of CPR, BLS using electrical defibrillation, ALS and post-CA care. ${ }^{9}$ However, few studies showed which factor would be the most important. In this research, we performed a retrospective cohort study using a database and medical records of patients with out-of-hospital cardiogenic (including suspected cardiogenic) CA transported by ambulance to our hospital. The objective of the research was to clarify what was the factor to have the relationship of good neurological outcome, based on outcome-related factors before hospital, and procedures/treatments performed after arrival at hospital using an Utstein Style template ${ }^{11}$ in patients with PCAS admitted to the intensive care unit (ICU).

\section{EMERGENCY MEDICINE SYSTEMS IN TOKYO, JAPAN}

When a 119 call is received, the Emergency Command Center in the Tokyo Fire Department (TFD) dispatches an emergency vehicle to the scene 
from the nearest fire station. Emergency services teams supplied by Tokyo's public services comprise three emergency services personnel who are usually on duty across a 24-hour schedule. At least one of these team members is a qualified emergency medical technician (EMT). EMTs can treat individuals experiencing CA at the scene, using BLS; securing an airway and providing a concentrated oxygen supply using bag-valve masks, laryngeal masks, oesophageal-tracheal combitubes (oesophageal obdurator airway) and tracheal tubes; securing a venous route for crystalloid solutions; intravenous administration of adrenaline; and defibrillation using an AED. Of these procedures, special procedures are defined as (1) securing an airway using equipment (oesophageal obdurator airway or laryngeal mask, securing an airway using a tracheal tube, and tracheal intubation by an individual accredited in this procedure); (2) securing a venous route and transfusion via a venous route using the lactate Ringer's solution and (3) drug administration using adrenaline (adrenaline administration by an individual accredited in drug administration). The box below shows the standards for performing special procedures at the scene by a EMT (box 1).

\section{PATIENTS AND METHODS}

\section{Patients and procedures}

This study was approved by the Clinical Research Institutional Review Board (IRB) of Nihon University School of Medicine

\section{Box 1}

Standards for first-aid activities in special procedures performed by emergency medical technicians Tokyo Fire Department First Aid Standards

Securing an airway

- Laryngeal mask, oesophageal obdurator airway double balloon tube

- In state of cardiac arrest (CA)

- Consciousness level Japan Coma Scale 300, respiratory function halted

- Tracheal tube

- In state of CA and has the following:

- Choking on a foreign object

- If another emergency attending physician deems it necessary

Transfusion by securing a peripheral venous route

- Patients for securing venous access

- Securing venous access is performed in the following case

- Injured/sick individual in a state of CA

- Comatose and with respiratory or cardiopulmonary arrest

- Methods for securing venous access

- Using a venous indwelling needle or a winged needle, puncture the site below, and transfuse lactate Ringer's solution

Pharmacotherapy

- Patient aged 8 years or older, or with a body weight of $25 \mathrm{~kg}$ or above, in a state of CA, and comply with the following;

- Injured/sick individual presenting with ventricular fibrillation or pulseless ventricular tachycardia.

- Injured/sick individual presenting with pulseless electrical activity

- Injured/sick individual presenting with asystole
Itabashi Hospital. Study subjects were out-of-hospital CA patients (including those suspected of cardiogenic CA) where the cause was cardiogenic (including suspected cardiogenic cases) and who had PCAS after ROSC. The study was designed as a single-institution retrospective cohort research. The study enrolled patients with PCAS transported to the Emergency and Critical Care Center of this hospital between 1 January 2008 and 31 December 2010. Data on the CA patients were tabulated in accordance with the Utstein Style, and aggregate analyses were performed on the basis of the hospitalisation register. These aggregate analyses were subjected to peer review through a conference between the emergency medicine specialists and cardiovascular specialists the day after the CA patient was transported to the hospital, evaluated using the Utstein Style, and the data populated into a database.

This study defines out-of-hospital CA patient as individuals where CA was confirmed at the scene by EMT after a 119 call was made. There are no clear standards governing the performance of coronary angiography after ROSC, but patients with suspected acute coronary syndrome based on ECG after ROSC, echocardiography and other tests, were tested using coronary angiography before being admitted to the ICU, and percutaneous coronary intervention (PCI) was performed in the vessels responsible.

In our hospital, therapeutic hypothermia $(\mathrm{TH})$ is indicated for cardiogenic (including suspected cardiogenic) CA patients in a coma after ROSC and on admission to the ICU. The temperature of the patients was maintained at $34^{\circ} \mathrm{C}$ for $24 \mathrm{~h}$ from the start of cooling. The patients were gradually rewarmed from $34^{\circ} \mathrm{C}$ to $36^{\circ} \mathrm{C}$ for $24 \mathrm{~h}$. Exclusion criteria for $\mathrm{TH}$ in PCAS are (1) unstable haemodynamics even with the use of vasopressors (mean blood pressure $<60 \mathrm{~mm} \mathrm{Hg}$ or systolic blood pressure $<90 \mathrm{~mm} \mathrm{Hg})$, (2) inadequate oxygenation $\left(\mathrm{PaO}_{2} / \mathrm{F}_{\mathrm{I}} \mathrm{O}_{2}\right.$ ratio $<200$ ), (3) patients in the end-stage of chronic disease and (4) where family consent is not given. ${ }^{12}$

Evaluation of the outcome from PCAS was performed when the patient was discharged or transferred from this hospital. The endpoint was whether a good neurological outcome was achieved or not. Neurological evaluation was performed using the Pittsburgh cerebral-performance categories (CPC). A good outcome was defined as CPC 1 (good recovery) or 2 (moderate disability), and a poor outcome was defined as CPC 3 (severe disability), 4 (vegetative state), or 5 (death). ${ }^{11}$

This research analysed the following factors related to outcome based on medical records and a CA patient database using the Utstein Style: (1) age, (2) gender, (3) witness present, (4) bystander CPR performed, (5) ECG waveform at the scene when emergency personnel arrived, (6) time interval from receipt of the 119 call to arrival of emergency personnel, (7) time interval from receipt of the 119 call to arrival at hospital, (8) prehospital defibrillation performed, (9) special procedures performed by EMTs and (10) time interval from receipt of the 119 call to ROSC. The following factors relating to treatment after hospitalisation were also investigated: (1) coronary angiography and PCI performed and (2) TH performed.

In order to compare factors related to neurological outcome in PCAS, the primary endpoint was defined as a good neurological outcome (CPC 1, 2) or poor neurological outcome (CPC $3,4,5)$ when patients with PCAS and admitted to the ICU were discharged or transferred from our hospital. For the secondary endpoint of surviving to hospital discharge, we compared surviving to hospital discharge (CPC 1, 2, 3, 4) and death (CPC 5).

\section{Statistical analysis}

Statistical analysis was performed using Stat-View V.5.0 (SAS Institute, Cary, North Carolina, USA; 1998). Student $t$ test was 


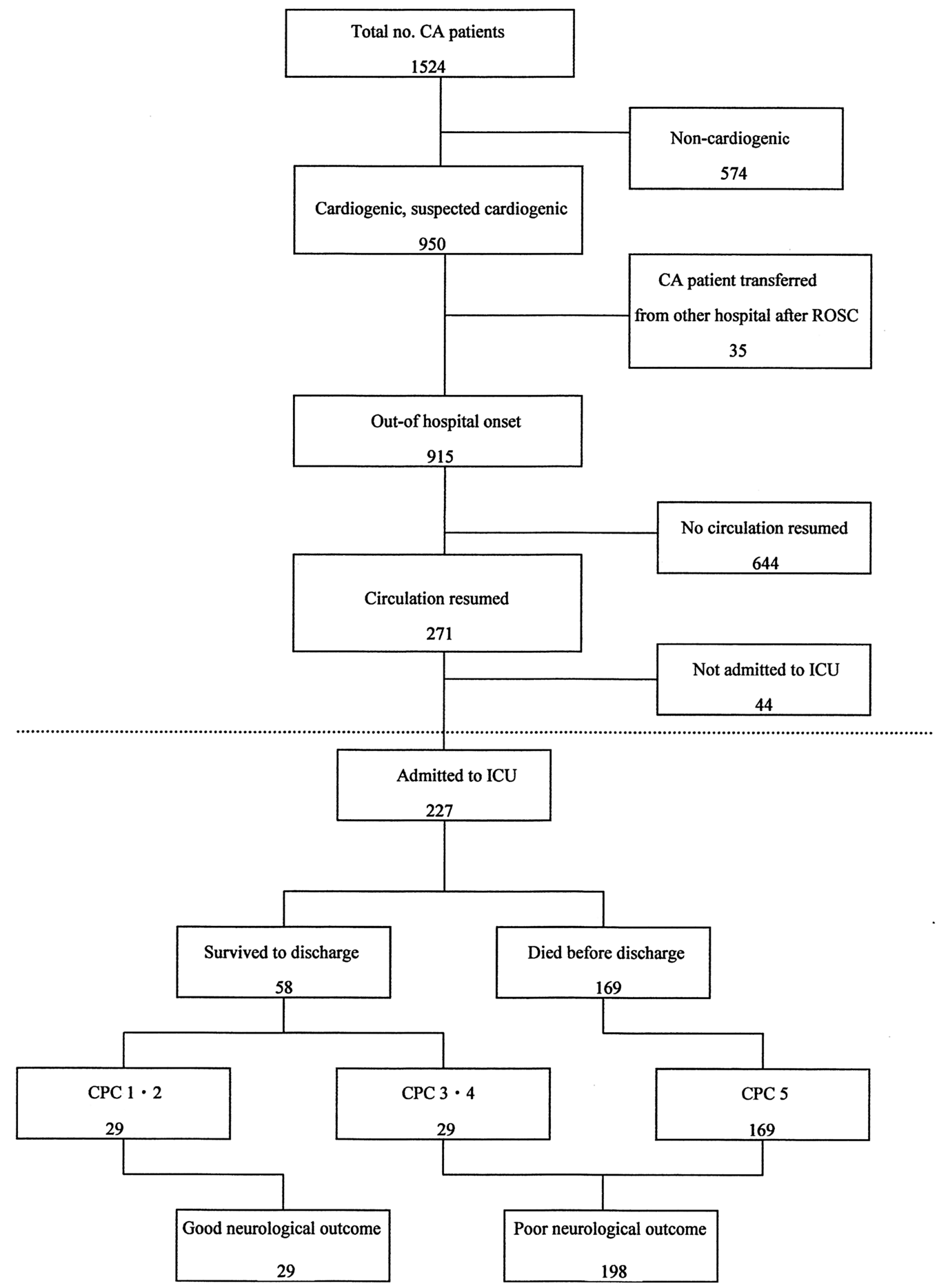

Figure 1 Breakdown of total numbers in patients with cardiopulmonary arrest. CA, cardiac arrest; CPC, Pittsburgh cerebral-performance categories; ICU, intensive care unit; ROSC, return of spontaneous circulation.

used to compare continuous variables, and Fisher's exact test was used to compare categorical data. Using these tests, we compared good and poor neurological outcome and compared survival and death. To predict outcome, we performed multiple logistic regression with a forced entry method, and calculated the ORs and 95\% CIs using the dependent variables of good or poor neurological outcome and survival or death, and the independent variables from the categorical data and the continuous variable data. The forced entry method was used for age and gender as explanatory variables, but these are not shown in the table. Data are expressed as mean $\pm \mathrm{SD}$ or as number of patients (\%). $\mathrm{p}<0.05$ Was defined as statistically significant.

\section{RESULTS}

There were a total of 1524 CA patients during the study period. Of these, 950 were cardiogenic (including suspected cardiogenic) CA patients. Excluding patients who suffered from CA inside a hospital or patients who were transported to this hospital after ROSC left 915 patients. A total of 271 patients achieved ROSC and 227 patients were admitted to the ICU 
Table 1 Comparison of factors related to neurological outcome in patients with postcardiac arrest syndrome

\begin{tabular}{|c|c|c|c|}
\hline & $\begin{array}{l}\text { CPC } 1 \cdot 2 \\
(\%)\end{array}$ & $\begin{array}{l}\text { CPC 3.4.5 } \\
(\%)\end{array}$ & $\begin{array}{l}p \\
\text { Value }\end{array}$ \\
\hline Total patients $(\mathrm{n})$ & 29 & 198 & \\
\hline Age & $58.7 \pm 14.2$ & $70.5 \pm 16.2$ & $<0.001$ \\
\hline Gender (male) & $26(89.7)$ & $133(67.2)$ & 0.016 \\
\hline Witness present & $26(89.7)$ & $118(59.6)$ & 0.002 \\
\hline Bystander CPR performed & $17(58.6)$ & $59(29.8)$ & 0.003 \\
\hline \multicolumn{4}{|l|}{ Initial ECG waveform } \\
\hline Ventricular fibrillation/PVT & $23(79.3)$ & $43(21.7)$ & $<0.001$ \\
\hline Asystole/pulseless electrical activity & $6(20.7)$ & $155(78.3)$ & \\
\hline \multicolumn{4}{|l|}{ Action at the scene } \\
\hline Defibrillation performed & $24(82.8)$ & $48(24.2)$ & $<0.001$ \\
\hline Special procedures performed & $9(31.0)$ & $51(25.7)$ & 0.652 \\
\hline \multicolumn{4}{|l|}{ Treatment } \\
\hline $\begin{array}{l}\text { Coronary angiography/PCI } \\
\text { performed }\end{array}$ & $18(62.1)$ & $48(24.2)$ & $<0.001$ \\
\hline $\begin{array}{l}\text { Therapeutic hypothermia } \\
\text { performed }\end{array}$ & $19(65.5)$ & $51(25.7)$ & $<0.001$ \\
\hline \multicolumn{4}{|l|}{ Time from 119 call (min) } \\
\hline Arrival at the scene & $6.4 \pm 2.5$ & $6.8 \pm 2.7$ & 0.557 \\
\hline Arrival at hospital & $33.6 \pm 6.4$ & $34.8 \pm 9.2$ & 0.479 \\
\hline ROSC & $18.3 \pm 15.1$ & $48.6 \pm 17.9$ & $<0.001$ \\
\hline
\end{tabular}

Special procedures refers to (1) securing an airway using equipment (oesophageal obdurator airway or laryngeal mask, securing an airway using a tracheal tube, and tracheal intubation by an individual accredited in this procedure); (2) securing a venous route and transfusion via a venous route using the crystalloid solution lactate Ringer's solution and (3) drug administration using adrenaline (adrenaline administration by an individual accredited in drug administration). CPC, cerebral-performance category, CPR, cardiopulmonary resuscitation; $\mathrm{PCl}$, percutaneous coronary intervention; PVT, pulseless ventricular tachycardia; ROSC, return of spontaneous circulation.

with PCAS (159 males, 68 females). Of the 227 patients admitted to the ICU with PCAS, 66 (29.1\%) were treated with PCI and $70(30.8 \%)$ were treated with TH. TH was not performed in 157 patients because of (1) improvements in the level of consciousness (11 patients), (2) unstable haemodynamics (142 patients), (3) inadequate oxygenation (2 patients), or
(4) end-stage chronic disease (2 patients). Of the 227 PCAS patients admitted to the ICU, $29(12.8 \%)$ achieved a good neurological outcome and $198(87.2 \%)$ had a poor neurological outcome (figure 1). Table 1 shows the characteristics of the PCAS patients with a good neurological outcome and with a poor neurological outcome. The good neurological outcome group of patients with PCAS at ICU was young, included many men, and included a statistically significant higher proportion of witnesses to the patient's collapse at the scene. A significantly higher $(p<0.001)$ number of patients where the initial ECG showed ventricular fibrillation (VF) or pulseless ventricular tachycardia (PVT) achieved a good neurological outcome. Of the prehospital care, there was a high proportion of patients receiving defibrillation after the 119 call and while being transported. However, there was no statistically significant difference in the proportion of patients receiving special procedures at the scene by EMTs. No statistically significant difference was seen in the time interval to the arrival of emergency personnel or the time interval from receipt of 119 call to arrival of the CA patient at hospital. The time interval from receipt of 119 call to ROSC was significantly shorter in the good neurological outcome group. Looking at the factors relating to the course after hospitalisation, the group with a good neurological outcome had a significantly higher proportion of patients receiving coronary angiography/PCI or TH. Of these prehospitalisation and posthospitalisation factors, analysis of the independent variables related to neurological outcome using multiple logistic regression showed that there was only a relationship with the time interval from receipt of the 119 call to ROSC; a longer time to ROSC (OR: 0.86 ; $95 \%$ CI 0.81 to 0.92 ; $\mathrm{p}<0.001$ ) was correlated with CPC 3, 4, 5 (poor neurological outcome) (table 2).

For the secondary endpoints, tables 3 and 4 show the results when the patients with PCAS admitted to the ICU were divided into two groups according to outcome: survival and death. There were significantly more survivors when the emergency personnel arrived quickly, and significantly more survivors when EMTs performed special procedures at the scene. Of these prehospital and posthospital factors, the independent factors related to surviving to hospital discharge that correlated to a good survival outcome were bystander CPR by a witness, special

Table 2 Factors related to a good neurological outcome in patients with postcardiac arrest syndrome

\begin{tabular}{|c|c|c|c|c|}
\hline Factors & $\mathrm{OR}$ and $95 \% \mathrm{Cl}$ & OR & $95 \% \mathrm{Cl}$ & p Value \\
\hline Witness present & $\begin{array}{ccc}\leftarrow \text { Poor outcome }(\mathrm{CPC} 3,4,5) & \text { Good outcome }(\mathrm{CPCl}, 2) & \overrightarrow{ } \\
0.01 & 0.1 & 1.0\end{array}$ & 3.83 & 0.50 to 29.62 & 0.198 \\
\hline Bystander CPR performed & \begin{tabular}{c|c|}
$0.01+1$ & 1 \\
\end{tabular} & 3.79 & 0.64 to 22.39 & 0.142 \\
\hline \multicolumn{5}{|l|}{ Initial ECG waveform } \\
\hline Ventricular fibrillation/PVT & & 14.00 & 0.96 to 204.60 & 0.054 \\
\hline \multicolumn{5}{|l|}{ Action at the scene } \\
\hline Defibrillation performed & & 0.46 & 0.03 to 7.37 & 0.579 \\
\hline Special procedures performed & \begin{tabular}{l|l}
+ \\
\end{tabular} & 2.31 & 0.46 to 11.52 & 0.309 \\
\hline \multicolumn{5}{|l|}{ Treatment } \\
\hline Coronary angiography/PCI performed & & 1.13 & 0.24 to 5.26 & 0.879 \\
\hline Therapeutic hypothermia performed & \begin{tabular}{l|l}
1 \\
\end{tabular} & 1.64 & 0.28 to 9.52 & 0.583 \\
\hline \multicolumn{5}{|l|}{ Time from 119 call (min) } \\
\hline Arrival at the scene & & 1.15 & 0.81 to 1.62 & 0.437 \\
\hline Arrival at hospital & + & 0.99 & 0.90 to 1.10 & 0.904 \\
\hline ROSC & + & 0.86 & 0.81 to 0.92 & $<0.001$ \\
\hline
\end{tabular}


Table 3 Comparison of factors related to survival and death in patients with postcardiac arrest syndrome

\begin{tabular}{|c|c|c|c|}
\hline & CPC 1.2.3.4 (\%) & CPC $5(\%)$ & p Value \\
\hline Total patients (n) & 58 & 169 & \\
\hline Age & $63.7 \pm 15.1$ & $70.8 \pm 16.5$ & 0.004 \\
\hline Gender (male) & $51(87.9)$ & $108(63.9)$ & $<0.001$ \\
\hline Witness present & $47(81.0)$ & $97(57.4)$ & 0.001 \\
\hline Bystander CPR performed & $31(53.4)$ & $45(26.6)$ & $<0.001$ \\
\hline \multicolumn{4}{|l|}{ Initial ECG waveform } \\
\hline Ventricular fibrillation/PVT & $39(67.2)$ & $27(16.0)$ & $<0.001$ \\
\hline Asystole/pulseless electrical activity & $19(32.8)$ & $142(84.0)$ & \\
\hline \multicolumn{4}{|l|}{ Action at the scene } \\
\hline Defibrillation performed & $44(75.9)$ & $28(16.6)$ & $<0.001$ \\
\hline Special procedures performed & $24(41.4)$ & $36(21.3)$ & 0.005 \\
\hline \multicolumn{4}{|l|}{ Treatment } \\
\hline Coronary angiography/PCI performed & $34(58.6)$ & $32(18.9)$ & $<0.001$ \\
\hline Therapeutic hypothermia performed & $39(67.2)$ & $31(18.3)$ & $<0.001$ \\
\hline \multicolumn{4}{|l|}{ Time from 119 call (min) } \\
\hline Arrival at the scene & $6.1 \pm 2.2$ & $6.9 \pm 2.8$ & 0.048 \\
\hline Arrival at hospital & $33.6 \pm 7.9$ & $35.0 \pm 9.2$ & 0.309 \\
\hline ROSC & $27.2 \pm 21.6$ & $50.8 \pm 15.7$ & $<0.001$ \\
\hline \multicolumn{4}{|c|}{$\begin{array}{l}\text { Special procedures refers to (1) securing an airway using equipment (oesophageal } \\
\text { obdurator airway or laryngeal mask, securing an airway using a tracheal tube, and } \\
\text { tracheal intubation by an individual accredited in this procedure); (2) securing a } \\
\text { venous route and transfusion via a venous route using the crystalloid solution lactate } \\
\text { Ringer's solution and (3) drug administration using adrenaline (adrenaline } \\
\text { administration by an individual accredited in drug administration). } \\
\text { CPC, cerebral-performance category, CPR, cardiopulmonary resuscitation; } \\
\text { PCl, percutaneous coronary intervention; PVT, pulseless ventricular tachycardia; } \\
\text { ROSC, return of spontaneous circulation. }\end{array}$} \\
\hline
\end{tabular}

procedures performed by a EMT, and a shorter time between receipt of the 119 call and ROSC (table 4).

\section{DISCUSSION}

In this research, as in previously reported studies, there were significantly more patients achieving a good neurological outcome even with PCAS where there were the prehospital factors of bystander CPR and VF/PVT on initial ECG and the posthospitalisation treatment-related factors of coronary angiography/PCI and TH after ROSC. However, of note in this study was the finding that while it is important for a witness to the event to perform bystander CPR and EMTs to perform special procedures in order to increase survival rates in patients with PCAS, this alone is insufficient to achieve a good neurological outcome. The key is how to reduce the time interval from receipt of the 119 call to ROSC.

Hayakawa $e t ~ a l^{13}$ reported that time from collapse to ROSC was one of the most important prognostic indicators for the patients after ROSC. The present study also showed the importance of ischaemic time interval for the patients with PCAS. Generally, the outcome for CA patients treated with TH is most strongly correlated with the time from collapse with CA to ROSC (median $25 \mathrm{~min}$ ), regardless of whether the initial ECG showed VF, asystole, or pulseless electrical activity. ${ }^{14}$ For neurological outcome, however, $57.9 \%$ of CA patients achieving ROSC in $25 \mathrm{~min}$ or less had a good neurological outcome, compared with zero patients with a good outcome where time to ROSC was over 25 min even with TH. The same study also showed that a good neurological outcome was only achieved in $21.2 \%$ of patients where the time to ROSC was 25 min or less but who were not treated with TH. This suggests that a good outcome is influenced by the length of time the patient experiences cerebral ischaemia during the CA and CPR.

The two cornerstone studies proved the efficacy of $\mathrm{TH}$ for PCAS patients. ${ }^{15} 16$ However, the present study showed that $\mathrm{TH}$ was not associated with a good neurological outcome. One of the inclusion criteria of these two studies was VF/PVT as the initial cardiac rhythm. ${ }^{15} 16$ In the present study, the criteria of TH included not only VF/PVT but also other non-shockable rhythm. For the PCAS patients with non-shockable as the initial cardiac rhythm, TH was reported not to be associated with good outcomes. ${ }^{17}$ The reason why $\mathrm{TH}$ was not an independent factor for good neurological outcomes in the present study may be the difference in the criteria for initial cardiac rhythm. The design of the present study could not elucidate the answer to the efficacy of TH. Further study is needed.

With the emergency medicine system in Japan, it can be unclear as to when precisely the CA occurred, and the patient transported to hospital even if a long time has passed since the CA. Similar results are obtained even in investigations restricted

Table 4 Factors related to survival in patients with postcardiac arrest syndrome

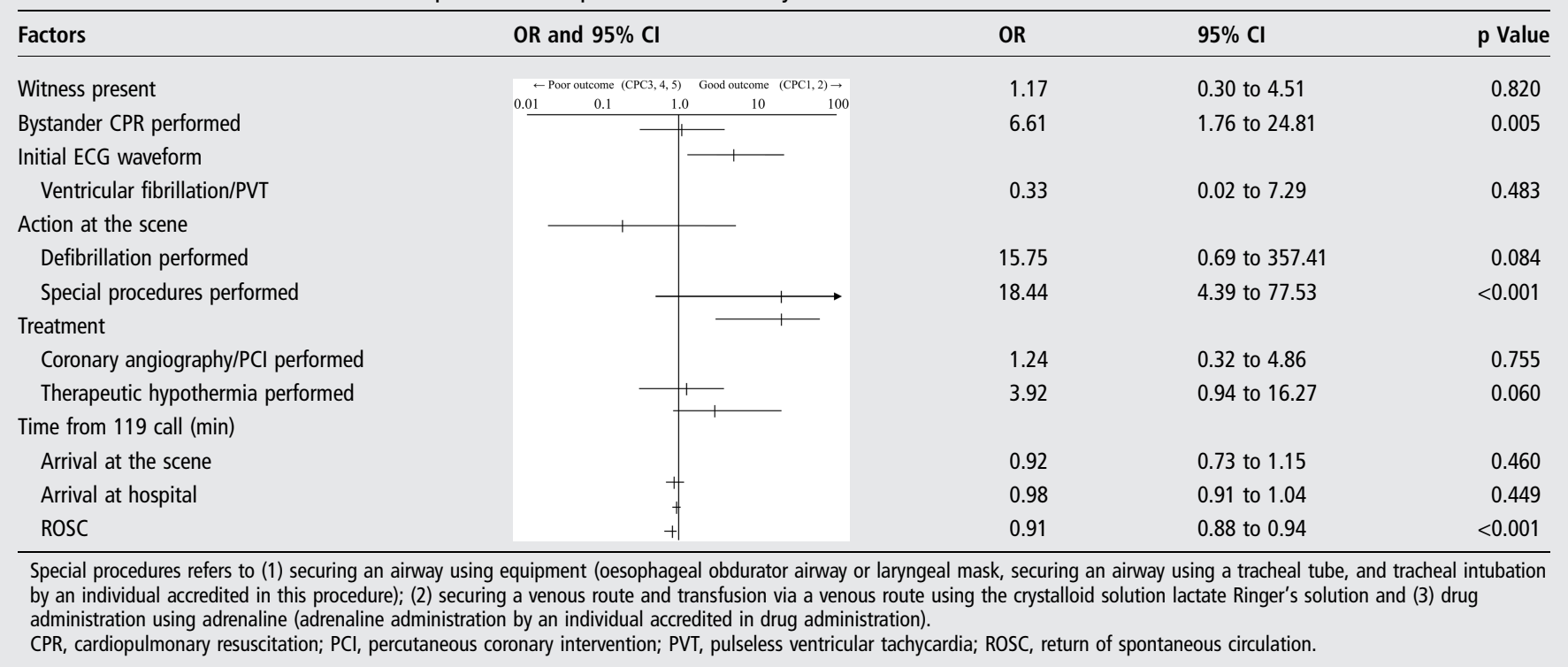


to only those patients with PCAS where there was a witness to the event. The Utstein Style ${ }^{11}$ emphasises the time that has passed since the emergency call, so this research also calculated the time to ROSC based on the time since the receipt of the 119 call rather than the time since the CA. The predicting score for survival consisted of immediate cause of CA, initial cardiac rhythm on ambulance arrival, presence of witnesses and bystander CPR and so on. ${ }^{18-20}$ However, ischaemic time interval was not included in these scores. The collapse time was not an exact time and would be presumed in many cases. This may be the reason why the time interval from collapse to ROSC was not included in these scores. In the present study we can show the effectiveness of the time interval from receipt of the 119 call to ROSC, to predict a good neurological outcome for PCAS patients, instead of the time interval from collapse to ROSC. Call receipt time is an official time, and is reliable. Therefore, we may use the time from receipt of the call to ROSC to create a predicting score for a good neurological outcome.

This study showed that survival rates for patients with PCAS are increased with bystander CPR and special procedures by EMTs, but the results suggested it may be even more important to try to reduce the time interval to ROSC if the patient is to be a good neurological outcome. Of particular interest is a study by Yasunaga et al that analysed data from Japan and showed that better outcomes can be achieved in more patients if bystander CPR is followed by ALS from physicians, rather than EMTs. According to Yasunaga et al, ${ }^{21}$ special procedures performed by EMTs before hospital increase survival rates but do not affect neurological outcome. His study supports the findings from another study by Stiell et al. ${ }^{4}$ Ambulances in Japan have two EMTs providing CPR while the patient is being transported to hospital, so there may be limits to the amount of continuous BLS or ALS they can provide inside the cramped vehicle and in a moving environment.

Cerebral blood flow during CPR is poor. Dogs with CA and with no CPR for 5 min had 41\% neurological deficit after resuscitation. $^{22}$ CPR immediately after CA appears to be an essential factor from the aspect of brain resuscitation. The present study suggests cerebral blood flow may be insufficiently restored for the brain in the case of prolonged chest compression in CPR, because of attaining wide extent from $4 \%$ to $60 \%$ of normal level at standard external chest compression. ${ }^{23}$ The brain may have a time-sensitive vulnerability for whole-brain ischaemia even though CPR is performed. In studies to investigate lowering the body temperature by emergency personnel administering an infusion solution cooled to $4^{\circ} \mathrm{C}$ to out-of-hospital CA patients either during $\mathrm{CPR}^{24}$ or after ROSC, ${ }^{25}$ these techniques were shown to be safe even during CPR and effective in producing hypothermia even after ROSC. These results suggest that in regions where it takes time to transport a patient to hospital, improved outcomes may be achieved through simple and effective methods to initiate $\mathrm{TH}$ at the scene when ROSC is achieved and thereafter.

\section{CONCLUSIONS}

Bystander CPR by a witness at the scene of the CA followed by special procedures performed by emergency personnel is important in increasing survival rates in patients with PCAS. However, these factors are insufficient if the goal is to achieve a good neurological outcome. A good neurological outcome requires a shorter time interval from receipt of the 119 call to ROSC. We should take measures to improve the emergency system for CA patients to achieve earlier ROSC, and should develop brain protection during $\mathrm{CA}$ at the prehospital scene.
Contributors TK, AS and KK contributed to the conception of this study. TK, TM, $J Y, A S, R K$ and KT contributed to the clinical performance. TK analysed and wrote this manuscript, and AS edited and revised it.

\section{Competing interests None.}

Ethics approval Clinical Research Institutional Review Board of Nihon University Itabashi Hospital.

Provenance and peer review Not commissioned; internally peer reviewed.

Open Access This is an Open Access article distributed in accordance with the Creative Commons Attribution Non Commercial (CC BY-NC 3.0) license, which permits others to distribute, remix, adapt, build upon this work non-commercially, and license their derivative works on different terms, provided the original work is properly cited and the use is non-commercial. See: http://creativecommons.org/ licenses/by-nc/3.0/

\section{REFERENCES}

1 Nolan JP, Neumar RW, Adrie C, et al. Post-cardiac arrest syndrome: epidemiology, pathophysiology, treatment, and prognostication. A Scientific Statement from the International Liaison Committee on Resuscitation; the American Heart Association Emergency Cardiovascular Care Committee; the Council on Cardiovascular Surgery and Anesthesia; the Council on Cardiopulmonary, Perioperative, and Critical Care; the Council on Clinical Cardiology; the Council on Stroke. Resuscitation 2008;79:350-79.

2 Neumar RW, Nolan JP, Adrie C, et al. Post-cardiac arrest syndrome: epidemiology, pathophysiology, treatment, and prognostication. A consensus statement from the International Liaison Committee on Resuscitation (American Heart Association, Australian and New Zealand Council on Resuscitation, European Resuscitation Council, Heart and Stroke Foundation of Canada, InterAmerican Heart Foundation, Resuscitation Council of Asia, and the Resuscitation Council of Southern Africa); the American Heart Association Emergency Cardiovascular Care Committee; the Council on Cardiovascular Surgery and Anesthesia; the Council on Cardiopulmonary, Perioperative, and Critical Care; the Council on Clinical Cardiology; and the Stroke Council. Circulation 2008;118:2452-83.

3 Carr BG, Kahn JM, Merchant RM, et al. Inter-hospital variability in post-cardiac arrest mortality. Resuscitation 2009;80:30-4.

4 Stiell IG, Wells GA, Field B, et al. Advanced cardiac life support in out-of-hospital cardiac arrest. N Engl J Med 2004;351:647-56.

5 Keenan SP, Dodek P, Martin C, et al. Variation in length of intensive care unit stay after cardiac arrest: where you are is as important as who you are. Crit Care Med 2007:35:836-41.

6 Mashiko K, Otsuka T, Shimazaki S, et al. An outcome study of out-of-hospital cardiac arrest using the Utstein template-a Japanese experience. Resuscitation 2002:55:241-6.

7 Wijdicks EF, Hijdra A, Young GB, et al. Quality Standards Subcommittee of the American Academy of $\mathrm{N}$. Practice parameter: prediction of outcome in comatose survivors after cardiopulmonary resuscitation (an evidence-based review): report of the Quality Standards Subcommittee of the American Academy of Neurology. Neurology 2006;67:203-10.

8 International Liaison Committee on R. 2005 International Consensus on Cardiopulmonary Resuscitation and Emergency Cardiovascular Care Science with Treatment Recommendations. Part 4: Advanced life support. Resuscitation 2005:67:213-47.

9 Travers AH, Rea TD, Bobrow BJ, et al. Part 4: CPR Overview: 2010 American Heart Association Guidelines for Cardiopulmonary Resuscitation and Emergency Cardiovascular Care. Circulation 2010;122:S676-84

10 Neumar RW, Otto CW, Link MS, et al. Part 8: adult advanced cardiovascular life support: 2010 American Heart Association Guidelines for Cardiopulmonary Resuscitation and Emergency Cardiovascular Care. Circulation 2010;122(18 Suppl 3):S729-67.

11 Cummins RO, Chamberlain DA, Abramson NS, et al. Recommended guidelines for uniform reporting of data from out-of-hospital cardiac arrest: the Utstein Style. A statement for health professionals from a task force of the American Heart Association, the European Resuscitation Council, the Heart and Stroke Foundation of Canada, and the Australian Resuscitation Council. Circulation 1991:84:960-75.

12 Sakurai A, Kinoshita K, Moriya T, et al. Reduced effectiveness of hypothermia in patients lacking the wave $V$ in auditory brainstem responses immediately following resuscitation from cardiac arrest. Resuscitation 2006;70:52-8

13 Hayakawa K, Tasaki 0, Hamasaki T, et al. Prognostic indicators and outcome prediction model for patients with return of spontaneous circulation from cardiopulmonary arrest: the Utstein Osaka Project. Resuscitation 2011;82:874-80.

14 Oddo M, Ribordy V, Feihl F, et al. Early predictors of outcome in comatose survivors of ventricular fibrillation and non-ventricular fibrillation cardiac arrest treated with hypothermia: a prospective study. Crit Care Med 2008;36:2296-301.

15 Hypothermia after Cardiac Arrest Study G. Mild therapeutic hypothermia to improve the neurologic outcome after cardiac arrest. N Engl J Med 2002;346:549-56.

16 Bernard SA, Gray TW, Buist MD, et al. Treatment of comatose survivors of out-ofhospital cardiac arrest with induced hypothermia. N Engl J Med 2002;346:557-63. 
17 Dumas F, Grimaldi D, Zuber B, et al. Is hypothermia after cardiac arrest effective in both shockable and nonshockable patients?: insights from a large registry. Circulation 2011:123:877-86.

18 Eisenberg $M$, Hallstrom A, Bergner L. The ACLS score. Predicting survival from out-of-hospital cardiac arrest. JAMA 1981;246:50-2.

19 Cooper S, Evans C. Resuscitation Predictor Scoring Scale for inhospital cardiac arrests. Emerg Med J 2003;20:6-9.

20 Sladjana A. A prediction survival model for out-of-hospital cardiopulmonary resuscitations. J Crit Care 2011;26:223 e11-18.

21 Yasunaga $\mathrm{H}$, Horiguchi $\mathrm{H}$, Tanabe $\mathrm{S}$, et al. Collaborative effects of bystander-initiated cardiopulmonary resuscitation and prehospital advanced cardiac life support by physicians on survival of out-of-hospital cardiac arrest: a nationwide population-based observational study. Crit Care 2010; 14:R199.

22 Sanders $A B$, Kern $K B$, Bragg $S$, et al. Neurologic benefits from the use of early cardiopulmonary resuscitation. Ann Emerg Med 1987;16:142-6.

23 Henry R. Halperin BKR. Manual cardiopulmonary resuscitation techniques. In: Paradis NA, Halperin HR, Kern KB, Wenzel V, Chamberlain DA, eds. Cardiac arrest. Cambridg: Cambridg University Press, 2007:571-84

24 Kamarainen A, Virkkunen I, Tenhunen J, et al. Prehospital induction of therapeutic hypothermia during CPR: a pilot study. Resuscitation 2008;76:360-3.

25 Kamarainen A, Virkkunen I, Tenhunen J, et al. Prehospital therapeutic hypothermia for comatose survivors of cardiac arrest: a randomized controlled trial. Acta Anaesthesiol Scand 2009:53:900-7. 